\title{
Diagnostics of Nanodisperse Polycrystals Based on the Polarization Bremsstrahlung of Relativistic Electrons
}

\author{
V. I. Alekseev ${ }^{a}$, A. N. Eliseev ${ }^{a}$, E. F. Irribarra ${ }^{b}$, P. M. Nazhmudinov ${ }^{b}$, N. N. Nasonov ${ }^{b}$, \\ A. S. Kubankin ${ }^{a, b}$, V. V. Polyanskii ${ }^{a}$, and V. I. Sergienko ${ }^{a}$ \\ ${ }^{a}$ Lebedev Physical Institute, Russian Academy of Sciences, Moscow, 119991 Russia \\ e-mail:kubankin@bsu.edu.ru,eliseev@pluton.lpi.troizk.ru,polyansk@sci.lebedev.ru, sergienk@x4u.lebedev.ru \\ ${ }^{b}$ Laboratory of Radiation Physics, National Research University Belgorod State University, Belgorod, 308015 Russia \\ e-mail:estebanirribarra@hotmail.com, fizeg@bk.ru,nnn@bsu.edu.ru \\ Received July 17, 2013
}

\begin{abstract}
The spectra of polarization bremsstrahlung are measured in the backscattering geometry during the interaction of 7-MeV electrons with a polycrystalline Ni foil. Measurement is conducted under conditions such that the size of the region of coherent X-ray radiation scattering in a target is on the order of $10 \mathrm{~nm}$. The obtained results make it possible to suggest that using polarization bremsstrahlung as a new method for the diagnostics of the atomic structures of nanodisperse polycrystals is effective.
\end{abstract}

DOI: $10.1134 / \mathrm{S} 1027451014020293$

\section{INTRODUCTION}

The polarization bremsstrahlung $(\mathrm{PB})$ of relativistic electrons $[1,2]$ can be used for the structure diagnostics of polycrystalline media on the basis of the energy-dispersion technique [3-8]. The potential advantages of the approach under discussion include precise knowledge of the spectrum of pseudophotons of the Coulomb field of fast electrons (the pseudophotons play the role of the primary probe radiation), which is required within the framework of the energydispersion technique, and also the possibility of obtaining high spatial-measurement resolution because of the simple magnetic focusing of an electron beam on a target [4]. The authors of [9] showed that it is possible to significantly increase the energy resolution of measurements in the scheme of BP peak recording in the direction opposite to the velocity of radiating electrons, and the authors of [7] were the first to reliably record this effect. The authors of [8] showed that it is possible to measure the BP signal in the backscattering geometry under the condition that the polycrystal grain size is smaller than the length of BP signal photoabsorption, which emphasized that the developed method for studying polycrystals with submicron grain size is well-grounded.An important problem determining the possibility of the use of PB as a new method for studying the atomic structures of polycrystalline media is measurement of the PB signal generated in polycrystals with a coherent-scattering-region size on the order of $10 \mathrm{~nm}$. This quantity is often encountered in material-science problems, and studying the structural char- acteristics of such polycrystals is one of the main problems of physical-material science.

In this paper, we record PB coherent peaks in the backscattering geometry for $7-\mathrm{MeV}$ electrons interacting with a Ni polycrystal in the case of a coherent scattering region with an average size of about $50 \mathrm{~nm}$. This target was made especially for the experiment and preliminarily studied using $\mathrm{X}$-ray analysis. The obtained results demonstrate that the developed method can be used to study polycrystal nanostructured media.

\section{EXPERIMENTAL}

PB in the backscattering geometry was studied experimentally using the setup [8] schematically shown in Fig. 1. The microtron 1 was used as the source of the electron beam. The $7-\mathrm{MeV}$ electron beam 2 was formed by carbon collimators 3 with an aperture of $5 \mathrm{~mm}$. The electron beam was transported to the target 7 which was fixed into the vacuum chamber 6 by means of three rotating magnets 4 , two pairs of magnetic quadrupole lenses 5 , and a corrector 6 , which made it possible to focus the electron beam on the place where the target was located with a size of up to $3 \mathrm{~mm}$ and with a divergence of, at most, $5 \mathrm{mrad}$. These values satisfied the PB measurement conditions in the backscattering geometry, which were limited by the dimensions of the transverse cross section of the electron beam and its divergence [7-9]. The transverse size, the position in the vacuum channel, and the current of the electron beam were controlled by the 


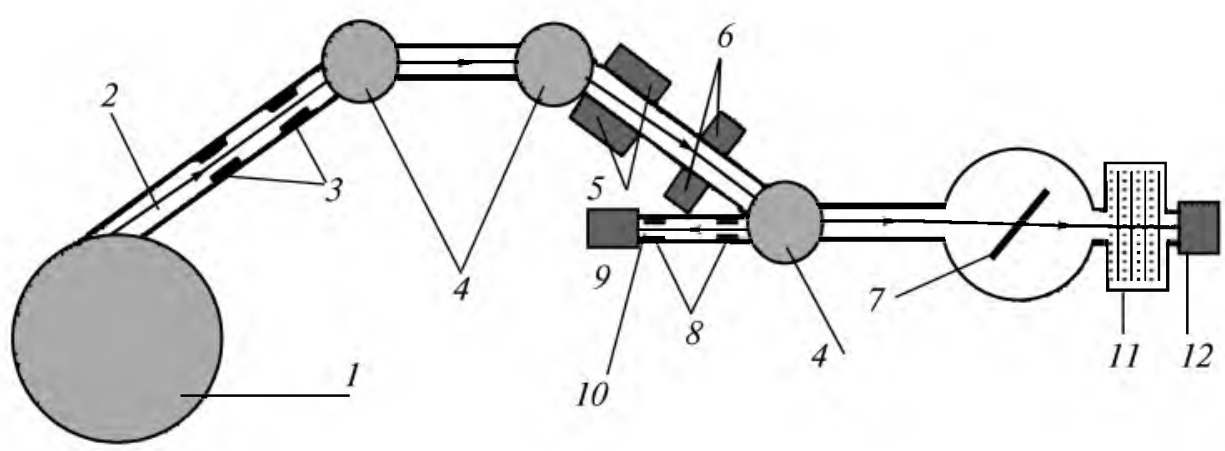

Fig. 1. Experimental setup.

proportional chamber 11 and a Faraday cylinder 12 . The PB signal 10 was formed by collimators 8 , whose dimensions were chosen so that the signal enters the detector 9 from the region of electron-beam-target interaction and partially from a target holder made of plexiglass. The collimators 8 were made of plexiglass; their apertures were 2 (for the collimator that was nearest to the detector) and $4 \mathrm{~mm}$. This peculiarity made it possible to exclude the background formed during the interaction of scattered electrons and X-ray radiation with setup components from the measured signal. The PB signal was recorded by an energy dispersion Si drift detector with an energy resolution of $130 \mathrm{eV}$ and a window area of $7 \mathrm{~mm}^{2}$. The rotating magnet 4 nearest to the target was placed so that it was possible to create the experiment geometry with the minimum background in the place where the detector was located; this background was produced by the microtron and collimators for the electron beam.

A Ni foil with a thickness of $40 \mu \mathrm{m}$ was used as the target. Preliminary studies performed using $\mathrm{X}$-ray analysis showed that the target has texture and that the average size of the backscattering region was about $50 \mathrm{~nm}$.

The target thickness was chosen from the condition that background radiation in the measurement region produced as a result of interaction of the electron beam with the proportional chamber and affecting the recorded signal form is absorbed. The detector was protected by $\mathrm{Pb}$ shielding from the external radiation background.

\section{MEASUREMENTS}

The setup was developed for measurements under conditions for the smallest manifestation of the background produced by electrons and $\mathrm{X}$-ray radiation in the spectrum of the measured PB signal in the form of peaks. For polycrystalline $\mathrm{Ni}$, the calculated positions of the PB spectral peaks in the backscattering geometry correspond to the following values: 3.05 (111),
$3.52(200), 4.97$ (220), 5.83 (311), 6.09 (222), and $7.03 \mathrm{keV}(400)$. The contribution of the characteristic $\mathrm{Ni}$ radiation $\left(K_{\alpha}\right.$ at $7.48 \mathrm{keV}$ and $K_{\beta}$ at $\left.8.26 \mathrm{keV}\right)$ was present in the signal together with the recorded $\mathrm{PB}$ peaks. The presence of the characteristic radiation peaks interferes with the PB spectrum measurements near these energy values within the limits of the detector energy resolution. Previously obtained results [5-8] showed the significant contribution of the escape peak to the measured spectrum of the PB signal. The escape peak is a phantom peak presented in the measured spectrum and formed as a result of the inelastic scattering of photons recorded by the Si detector. The calculated positions of the escape peaks produced during inelastic scattering of the Ni characteristic lines $K_{\alpha}$ and $K_{\beta}$ correspond to energies of 5.74 and $6.52 \mathrm{keV}$. Thus, it was possible to record freely only the PB peaks corresponding to the (111), (200), and (220) planes; the region where the other peaks are manifested overlaps with the characteristic radiation peaks and the escape peaks.

The X-ray study of the target showed the presence of the predominant orientation $[110]$ of the target surface plane; therefore, the PB spectrum was first measured under the condition of normal orientation of the target surface with respect to the electron beam. As a result, the PB peak corresponding to the (220) plane at an energy of $4.97 \mathrm{keV}$ was reliably recorded (Fig. 2). The spectrum was cut in a harder region at the beginning of the appearance of the peak of the Ni $K_{\alpha}$ line characteristic radiation, whose amplitude exceeds that of the PB signal by two orders or more. The lack of other peaks can be explained by the presence of texture, which was confirmed by recording the PB peaks corresponding to the (111) and (200) planes as the orientation angle was varied. Figure 3 shows the PB spectrum measured at an angle of $18^{\circ}$ between the normal to the target surface plane and the signal collimation axis (the angle was varied in the horizontal plane). 


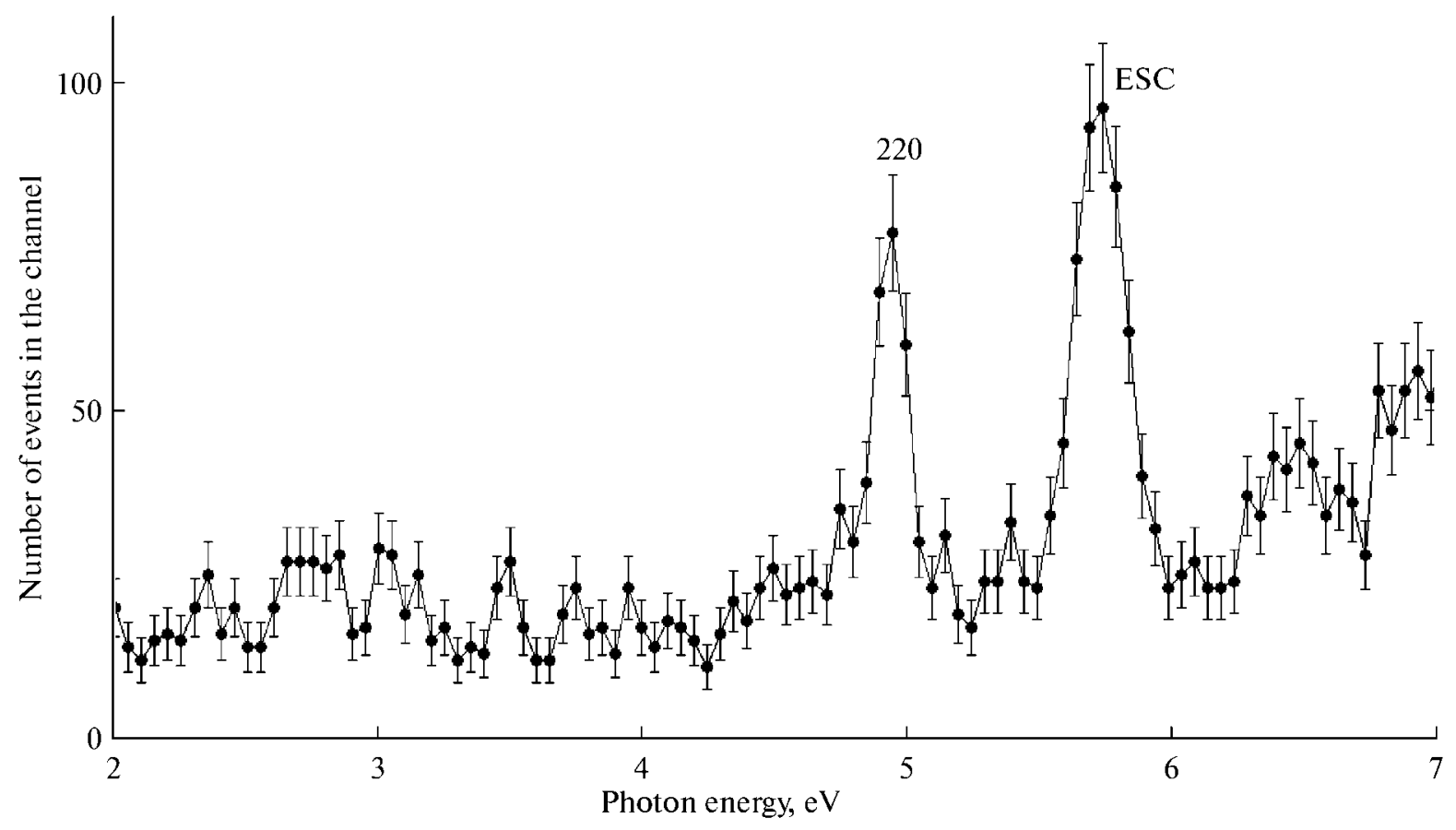

Fig. 2. Spectrum of "inverse" PB for a Ni polycrystal; the signal collimation axis is perpendicular to the target surface plane.

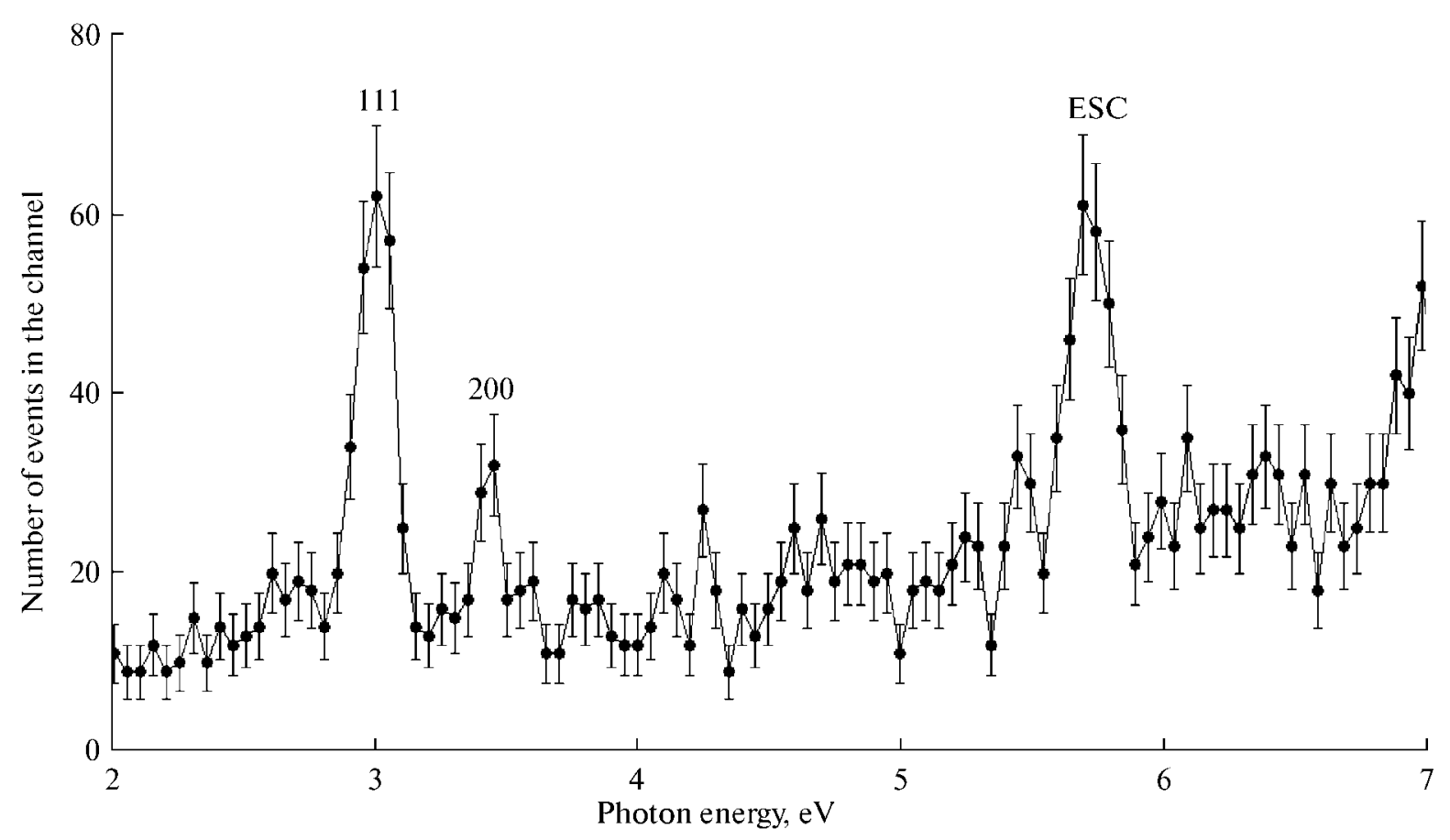

Fig. 3. Spectrum of "inverse" PB for a Ni polycrystal; the orientation angle is $18^{\circ}$.

\section{CONCLUSIONS}

Measurement of the PB spectrum generated in a polycrystalline $\mathrm{Ni}$ foil in the backscattering geometry in the case of the backscattering region with an average size of $50 \mathrm{~nm}$ showed the presence of coherent peaks whose positions agree well with the calculated data. The peaks corresponding to the crystallographic (111), (200), and (220) planes were reliably recorded for different target orientations with respect to the electron beam. It was difficult to identify peaks corresponding to the other planes because of texture. The obtained 
result demonstrates that a new developed method based on measuring the PB spectrum in the backscattering geometry and intended for studying the atomic structures of nanostructured polycrystalline media is promising.

\section{ACKNOWLEDGMENTS}

This work was supported by the Russian Foundation for Basic Research (grant no. 12-02-31389mol_a).

\section{REFERENCES}

1 1. M. Ya. Amus'ya, V. M. Buimistrov, and B. A. Zon, Polarization Bremsstrahlung of Particles and Atoms (Nauka, Moscow, 1987) [in Russian].

2. A. V. Korol', A. G. Lyalin, and A. V. Solov'ev, Polarization Bremsstrahlung (SPb. Gos. Ped. Univ., St.-Petersburg, 2004) [in Russian].

3. N. N. Nasonov, Nucl. Instrum. Methods Phys. Res, B 145, 19 (1998).
4. P. N. Zhukova, A. S. Kubankin, N. N. Nasonov, and V. I. Sergienko, Zavod. Labor., No. 10, 32 (2008).

5. V. A. Astapenko, A. S. Kubankin, N. N. Nasonov, V. V. Polyanskii, G. P. Pokhil, V. I. Sergienko, and V. A. Khablo, JETP Lett. 84, 281 (2006).

6. N. A. Gostishchev, A. S. Kubankin, N. N. Nasonov, V. V. Polyanskii, V. I. Sergienko, and V. A. Khablo, Tech. Phys. Lett. 34, 763 (2008).

7. V. I. Alekseev, K. A. Vokhmyanina, A. N. Eliseev, P. N. Zhukova, A. S. Kubankin, R. M. Nazhmudinov, N. N. Nasonov, V. V. Polyanskii, and V. I. Sergienko, Tech. Phys. Lett. 38, 294 (2012).

8. V. I. Alekseev, E. F. Irribarra, A. S. Kubankin, R. M. Nazhmudinov, N. N. Nasonov, V. V. Polyanskii, and V. I. Sergienko, J. Surf. Invest.: X-Ray, Synchrotron Neutron Tech. 7, 271 (2013).

9. V. Astapenko, N. N. Nasonov, and P. N. Zhukova, J. Phys. B: At. Mol. Opt. Phys. 40, 1 (2007).

Translated by L. Kul'man 